Revista Calidad en la Educación Superior

Programa de Autoevaluación Académica

Universidad Estatal a Distancia, Costa Rica

ISSN 1659-4703

revistacalidad@uned.ac.cr

\title{
EXPERIENCIA DE LA ASIGNATURA DE BASES DE DATOS CON LA CONSTRUCCIÓN DEL SISTEMA WEB PERSONALIZADO SUAEB. CASO DE ESTUDIO
}

\author{
EXPERIENCE OF THE SUBJECT DATABASE WITH CUSTOM \\ BUILDING SYSTEM WEB SUAEB. STUDY CASE
}

Ricardo-Adán Salas-Rueda ${ }^{1}$ adansalas@hotmail.com Universidad La Salle, D.F; México

\author{
Volumen 6, Número 2 \\ Noviembre 2015 \\ pp. $23-44$
}

Recibido: 13 de marzo, 2015

Aprobado: 21 de setiembre, 2015

\footnotetext{
1 Ricardo Adán Salas Rueda Doctor en Diseño de Nuevas Tecnologías, egresado de la Universidad Autónoma Metropolitana (UAM) en el año 2014. Durante 13 años ha impartido diversos cursos relacionados con la tecnología, la administración, las matemáticas en el nivel de licenciatura y posgrado. Estudió la Maestría en Administración de Empresas y la Ingeniería en Sistemas Electrónicos. Universidad La Salle, D.F., Universidad del Valle de México, D.F., Universidad da Vinci, México. Correo: adansalas@hotmail.com,
} 


\title{
Resumen
}

En la actualidad, los docentes junto con las universidades enfrentan el reto de perfeccionar las condiciones educativas que prevalecen en el salón de clases. La tecnología de punta y el diseño instruccional permiten la creación de nuevos entornos virtuales que apoyan el proceso de enseñanza-aprendizaje. Uno de los desafíos que enfrentan los docentes en la actualidad es facilitar la asimilación y utilización del conocimiento en el contexto laboral. Por esta razón, esta investigación propone la construcción del Sistema Usable de Aprendizaje-Enseñanza sobre Bases de Datos (SUAEB) para mejorar el proceso educativo a través de la personalización de los contenidos audiovisuales. Este sistema web ofrece a los estudiantes 128 contenidos audiovisuales que se adaptan a las necesidades del usuario. Además esta investigación se apoya en el enfoque cuantitativo para analizar el comportamiento de 19 estudiantes que utilizan el SUAEB durante la impartición del curso presencial 2014-2 denominado Base de datos. Por medio del método ANOVA con los niveles de significancia de 0.05 y 0.01 , este estudio analiza el impacto de utilizar el SUAEB como apoyo didáctico en una universidad ubicada en el Distrito Federal, México.

Palabras clave: Tecnología; Educación; Usabilidad; Contenidos audiovisuales

\begin{abstract}
Currently, teachers with universities face the challenge of improving educational conditions in the classroom. The technology and instructional design allow the creation of new virtual environments that support the teaching-learning process. One of the challenges facing teachers today is to facilitate the assimilation and use of knowledge in the employment context. For this reason, this research proposes the construction of Usable Learning System Instruction on Databases (SUAEB) to enhance the educational process through customization of audiovisual content. This web system offers students 128 audiovisual content to fit user needs. Furthermore, this research is based on the quantitative approach to analyze the behavior of 19 students using the SUAEB for the delivery of classroom course called Database 2014-2. Through ANOVA with significance levels of 0.05 and 0.01 , this study analyzes the impact of using the SUAEB as didactic support in a university located in Mexico City.
\end{abstract}

Keywords: Technology, Education, Usability, Audiovisual contents 


\section{Introducción}

Actualmente, el contexto educativo de México está sufriendo constantes transformaciones debido a las demandas de la sociedad del siglo XXI y los avances de la tecnología de vanguardia relacionada con las plataformas virtuales. Como lo explican Hepp, Prats y Holgado (2015), los nuevos escenarios de aprendizaje presentan una perspectiva transformadora que incluye la formación de los educadores en relación con la tecnología donde se incorporan innovaciones pedagógicas y proyectos digitales abiertos, flexibles, creativos, reales y participativos. Diversas universidades en México han decidido capacitar a los docentes en los aspectos relacionados con el uso de la informática en el aula, el diseño instruccional y el desarrollo de las competencias con el propósito de utilizar nuevas herramientas tecnológicas y pedagógicas durante el proceso de enseñanza-aprendizaje. De hecho, Vázquez (2015) menciona que una de las tendencias globales que está influyendo en la educación superior es el componente online, el cual involucra el uso de la red, internet y las nuevas tecnologías para personalizar el conocimiento. El docente encargado de la asignatura de Bases de Datos se ha dado cuenta que los estudiantes presentan dificultades para entender y aplicar los contenidos teóricos en el campo laboral. De acuerdo con Salaverría (2005), el gran reto de la construcción de los sistemas web educativos en el Siglo XXI consiste en considerar el aspecto de la usabilidad. Por esta razón, esta investigación considera a la usabilidad y la accesibilidad de los contenidos audiovisuales como los elementos fundamentales para la construcción de las plataformas web con el objetivo de facilitar la asimilación y reconstrucción del conocimiento. Por consiguiente, en el año 2014 se construyó el Sistema denominado SUAEB para presentar diversos contenidos audiovisuales, los cuales se adaptan a las necesidades y demandas de los estudiantes que cursan la asignatura de Bases de datos.

El objetivo general de esta investigación es 
- Diseñar e implementar el SUAEB en la asignatura de bases de datos correspondiente al ciclo escolar 2014-2 en la carrera de Informática Administrativa

A continuación se mencionan los elementos y criterios de la usabilidad, los principios de la accesibilidad de los contenidos audiovisuales y los conceptos sobre las bases de datos utilizados en esta investigación para la construcción del SUAEB.

\section{Metodología}

Este estudio se apoya en el enfoque cuantitativo con la finalidad de analizar el comportamiento de 19 estudiantes que cursan la asignatura de Bases de datos durante el ciclo 2014-2 en una universidad ubicada en el Distrito Federal, México. De acuerdo con Anderson, Sweeney y Williams (2011), el método ANOVA o Análisis de la varianza es un procedimiento estadístico que permite analizar los efectos de diferentes factores en el diseño de experimentos.

Esta investigación por medio del Método ANOVA analiza el aprovechamiento académico que presentan los estudiantes antes y después de utilizar el SUAEB en el proceso de enseñanza-aprendizaje. Por consiguiente, la variable independiente está relacionada con el uso del SUAEB y la variable dependiente son las calificaciones obtenidas por los estudiantes. Para ello, se utilizaron dos cuestionarios para analizar el comportamiento académico de los estudiantes antes (pre_prueba) y después (post_prueba) de utilizar el SUAEB.

Las hipótesis para esta investigación son:

Hipótesis Nula (Ho): Los estudiantes que utilizaron el SUAEB no mejoraron su rendimiento académico sobre las bases de datos 
Hipótesis Alternativa (Ha): Los estudiantes que utilizaron el SUAEB mejoraron su rendimiento académico sobre las bases de datos.

\section{Usabilidad}

La usabilidad facilita la transmisión de la información en la web por medio del uso adecuado de la estética, la accesibilidad de los contenidos audiovisuales, el diseño instruccional y el análisis de las características de las personas. De acuerdo con Brinck, Gergie y Wood (2002), la organización de los entornos virtuales usables permite la funcionalidad correcta, el uso eficiente, la facilidad de aprender y recordar, el error a la tolerancia y la sensación placentera en el uso de la interfaz Web. En esta investigación, el SUAEB propone la personalización de los contenidos audiovisuales relacionados con las bases de datos para que los estudiantes adquieran el conocimiento que necesitan de acuerdo a sus expectativas, habilidades y características.

Granollers, Lorés y Cañas (2005) explican que la ISO 9241 define a la usabilidad como "la medida en la que un producto puede ser usado por determinados usuarios para conseguir objetivos específicos con efectividad, eficiencia y satisfacción en un contexto de uso especificado" (p. 96).

Asimismo Gross (2011) menciona: "la usabilidad es la eficacia, eficiencia y satisfacción con la que un producto permite alcanzar objetivos específicos a usuarios específicos en un contexto de uso específico" (p.100)

La eficacia se refiere a la adquisición de información útil y la facilidad para localizar los datos, la eficiencia está asociada con el tiempo que emplea un individuo para lograr las metas y la satisfacción permite conocer si el diseño de la interfaz web cumple con las expectativas del usuario final. 
Según Suarez (2011), existen diversos estándares internacionales relacionados con el aspecto de la usabilidad como:

- ISO/IEC 9126 : es utilizado para la evaluación de la calidad del software compuesto por la funcionalidad, fiabilidad, usabilidad, eficiencia y portabilidad

- ISO/DIS9241-11 : está enfocado a la calidad en la usabilidad y ergonomía tanto de hardware como de software

- ISO 13407: sirve de guía para conseguir el desarrollo de sistemas interactivos usables durante el ciclo de vida del desarrollo

- ISO TR 18529: establece el proceso de ciclo de vida centrado en el usuario

- ISO TR 16982: explica los métodos de soporte de diseños centrados en usuarios

- ISO 9241-151: provee una guía del diseño centrado en el usuario relacionado con el software en la web

En la tabla 1 se presenta las principales características de estos estándares internacionales vinculados con la usabilidad.

Tabla 1. Estándares internacionales de la usabilidad

\begin{tabular}{|c|c|c|c|c|c|}
\hline ISO/IEC 9126 & ISO/DIS9241 & ISO 13407 & ISO TR 18529 & ISO TR 16982 & ISO 9241-151 \\
\hline $\begin{array}{l}\text { Comprensibilidad } \\
\text { (propósito) }\end{array}$ & $\begin{array}{l}\text { Eficacia } \\
\text { (exactitud) }\end{array}$ & $\begin{array}{l}\text { Diseño } \\
\text { centrado en el } \\
\text { usuario } \\
\text { (necesidades) }\end{array}$ & $\begin{array}{l}\text { Diseño } \\
\text { centrado en el } \\
\text { usuario } \\
\text { (necesidades) }\end{array}$ & $\begin{array}{l}\text { Métodos } \\
\text { ergonómicos }\end{array}$ & $\begin{array}{l}\text { Estrategia de } \\
\text { diseño }\end{array}$ \\
\hline $\begin{array}{l}\text { Aprendizaje } \\
\text { (documentación } \\
\text { y ayuda) }\end{array}$ & $\begin{array}{l}\text { Satisfacción } \\
\text { (confort y } \\
\text { aceptación) }\end{array}$ & $\begin{array}{l}\text { Factores } \\
\text { humanos }\end{array}$ & Planificación & $\begin{array}{l}\text { Diseño } \\
\text { centrado en el } \\
\text { usuario } \\
\text { (necesidades) }\end{array}$ & $\begin{array}{l}\text { Diseño del } \\
\text { contenido }\end{array}$ \\
\hline $\begin{array}{l}\text { Operabilidad } \\
\text { (personalización) }\end{array}$ & $\begin{array}{l}\text { Eficiencia } \\
\text { (recurso de } \\
\text { tiempo, }\end{array}$ & $\begin{array}{l}\text { Ergonomía } \\
\text { (optimización } \\
\text { de los }\end{array}$ & Requisitos & & $\begin{array}{l}\text { Navegación y } \\
\text { búsqueda }\end{array}$ \\
\hline Atractividad & $\begin{array}{l}\text { humanos y } \\
\text { financieros) }\end{array}$ & sistemas) & Producir & & $\begin{array}{l}\text { Presentación } \\
\text { del contenido }\end{array}$ \\
\hline
\end{tabular}

Fuente: Suárez (2011) 
De acuerdo con Rubin y Chisnell (2008), la usabilidad está relacionada con los siguientes aspectos:

- Útil: grado en que el usuario alcanza sus objetivos

- Eficiencia: rapidez con la que el usuario cubre sus necesidades

- Eficacia: funcionalidad del sistema

- Aprendizaje: habilidad del usuario para manejar el sistema

- Satisfacción: percepción del usuario sobre el uso del sistema

Para cumplir con los criterios de la usabilidad mencionados, el diseño de la arquitectura del SUAEB muestra al usuario un cuestionario que consta de 20 preguntas relacionado con los temas de las bases de datos con el propósito de presentar los contenidos audiovisuales que necesita el estudiante. La figura 1 muestra el módulo de adaptación que emplea el SUAEB.

Figura 1. Módulo de Adaptación del SUAEB. Diseño propio, 2015.

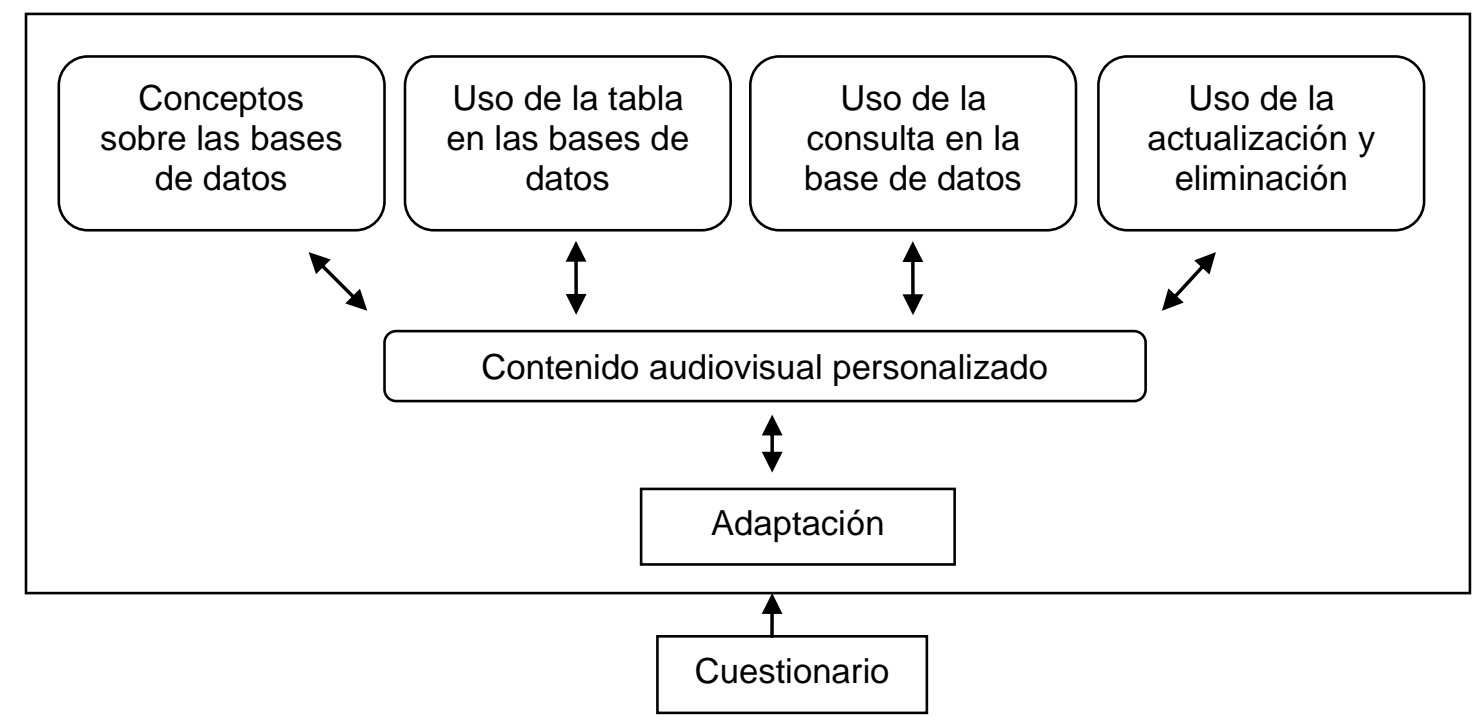

Fuente: Diseño propio, 2015. 
En la figura 1 se muestra que el módulo de adaptación del SUAEB cuyo propósito es construir el contenido audiovisual personalizado que necesita el estudiante considerando los aspectos relacionados con:

- Conceptos sobre las bases de datos

- Uso de la tabla en la base de datos

- Uso de la consulta en la base de datos

- Uso de la actualización y eliminación en la base de datos

González y Blanco (2012) mencionan que "la adaptación debe entenderse como la capacidad del sistema para que ajuste dinámicamente su conducta a los requerimientos de la interacción estudiante-sistema" (p.65). En particular, Kazanidis y Satratzemi (2007) establecen que la adaptación de los contenidos se refiere a la presentación dinámica de las páginas Web (texto, audio, sonido, video y animaciones) considerando el nivel de conocimiento, los objetivos y las características del usuario. Asimismo Lawrence y Tavakol (2007) mencionan que la metodología del diseño web constan de tres pilares: usabilidad, objetivo y estética.

Bernárdez (2007) describe los cinco factores relacionados con la usabilidad de Tara King:

- Utilidad: ¿la herramienta cumple eficazmente con la tarea?

- Apoyo a tarea: ¿el diseño ayuda a realizar la tarea más eficientemente?

- Adaptación al usuario: ¿puede ser usada en diferentes contextos?

- Adopción: ¿es preferida por los usuarios a otras alternativas?

- Extensibilidad ¿se puede usar para otras tareas no previstas?

De acuerdo con Kendall y Kendall (2011), el diagrama denominado Casos de uso provee a los desarrolladores un panorama sobre lo que desean los individuos. Esta investigación se apoya en este diagrama para identificar las necesidades (contenidos personalizados) que demanda el usuario respecto al uso de las bases de datos con la 
finalidad de determinar los criterios de usabilidad sobre el apoyo a la tarea y adaptación (figura 2).

Figura 2. Diagrama de caso de usos para el SUAEB.

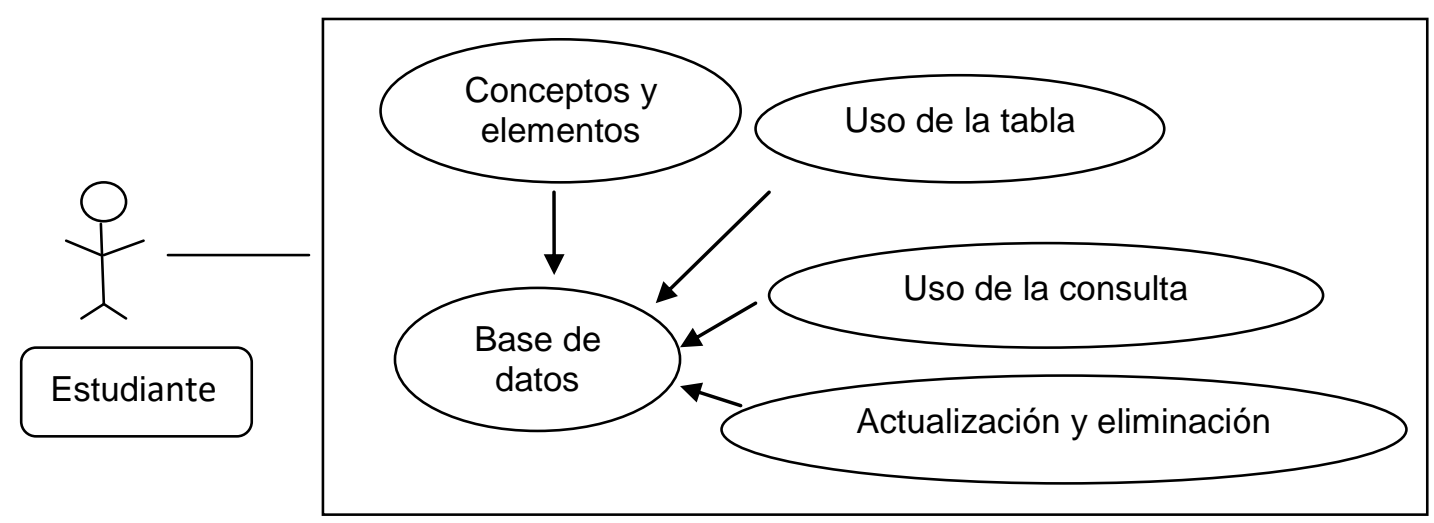

Fuente: Diseño propio, 2015.

.A continuación se describen los elementos y conceptos relacionados con la asignatura de base de datos que son utilizados durante el diseño de los contenidos audiovisuales del SUAEB.

\section{Contenidos de la asignatura Base de datos}

Hoy en día, las empresas invierten en diversos sistemas que utilizan las bases de datos para mejorar los procesos y las actividades productivas. De acuerdo con León (2008), el ERP (Enterprise Resource Planning) mejora la integración del negocio, facilita la planeación y el análisis para la toma de decisiones por medio del uso de la base de datos. Llanos (2007) explica que "una base de datos es un conjunto, colección o depósito de datos almacenados en un soporte informático de acceso directo. Los datos deben estar relacionados y estructurados de acuerdo con un modelo capaz de recoger el contenido semántico de los datos almacenados" (p. 272). 
Pons, Marín, Medina, Acid y Vila (2005) establecen que las ventajas de las bases de datos son:

- el mejoramiento de la organización de la información

- el control centralizado

- la escalabilidad de los sistemas

- el rendimiento del procesamiento

- la información integra

Las bases de datos utilizan el Lenguaje SQL (Structure Query Language) para administrar la información, es decir, los usuarios actualizan, insertan, borran y consultan los datos. Ramos y Ramos (2007) explican que el Lenguaje SQL es una herramienta para organizar, gestionar y recuperar la información almacenada en una base de datos relacional a través de la manipulación y definición de la información, el control de acceso y la administración de las transacciones. Los elementos y conceptos relacionados con las bases de datos como la tabla, las consultas y el Lenguaje SQL son indispensables durante la construcción de los sistemas.

La figura 3 muestra los elementos que son utilizados por el SUAEB para adaptar el contenido audiovisual de acuerdo a las necesidades del estudiante. 
Figura 3. Contenidos audiovisuales del SUAEB.

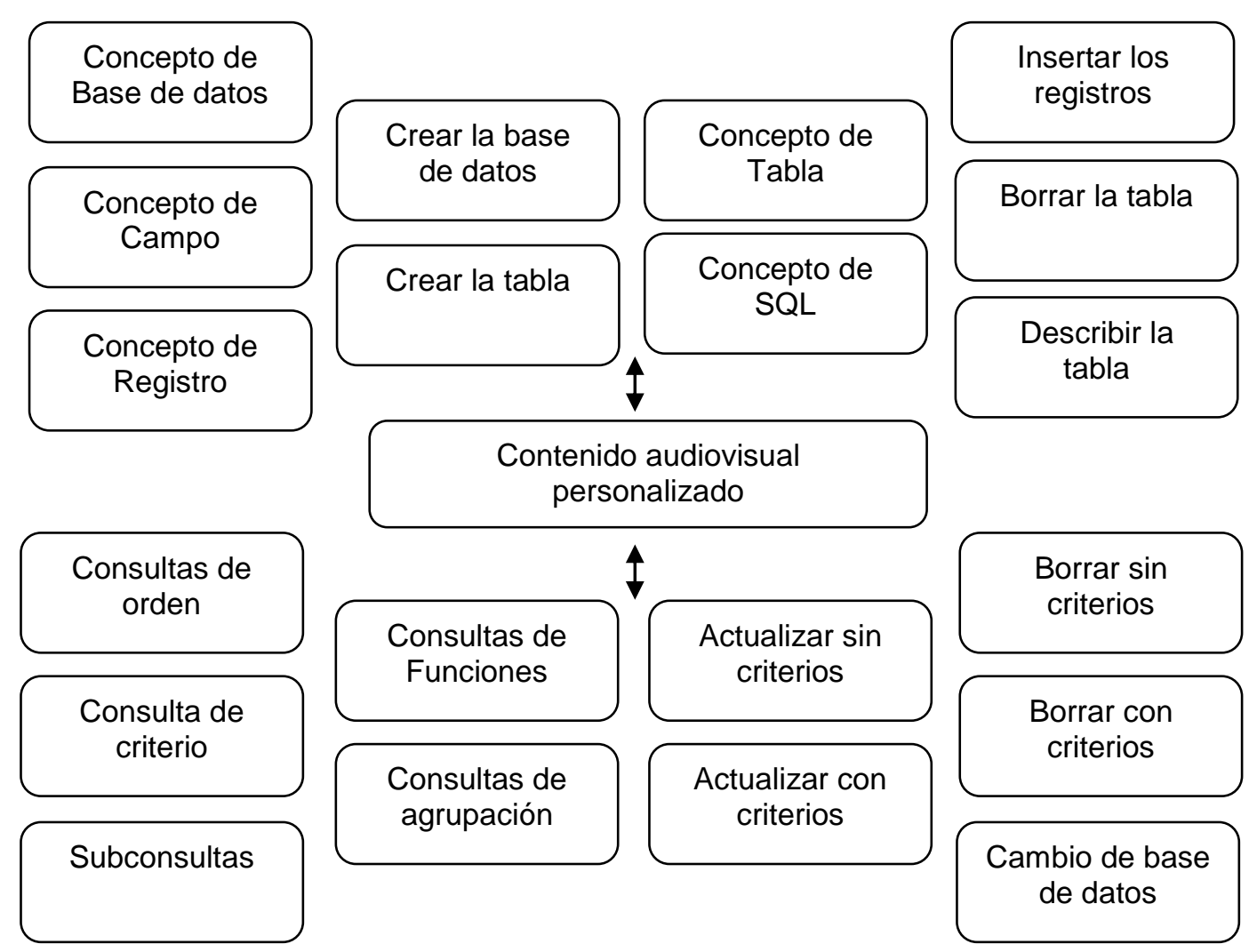

Fuente: Diseño propio, 2015.

Cabe mencionar que esta investigación propone la adaptación de los contenidos audiovisuales considerando el nivel de conocimiento que poseen los estudiantes por medio del SUAEB. 
La figura 4 muestra un ejemplo de los contenidos audiovisuales del SUAEB.

Figura 4. Contenidos audiovisuales del SUAEB. Diseño propio, 2015.

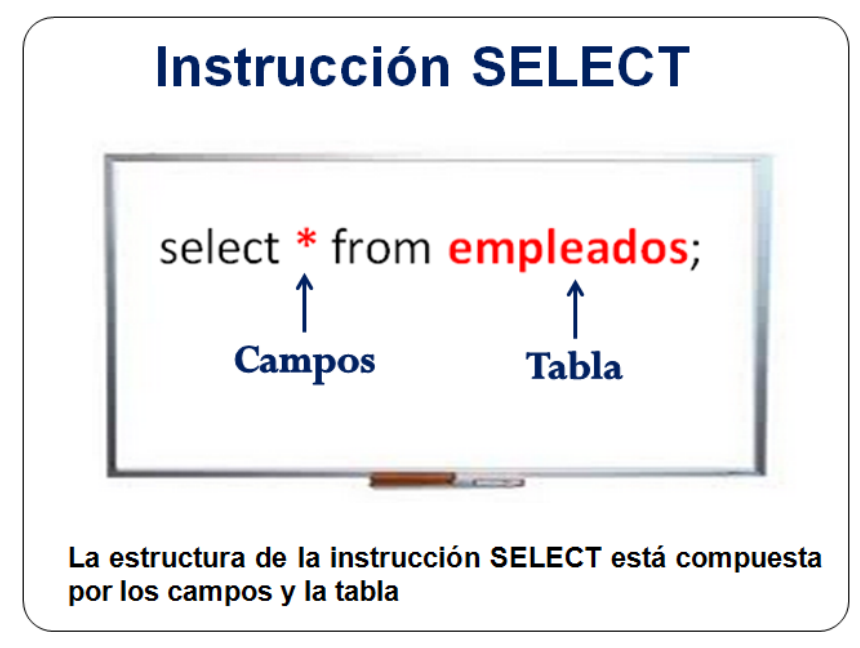

Fuente: Diseño propio, 2015.

Durante el diseño de los contenidos audiovisuales se consideró el aspecto de la accesibilidad.

\section{Accesibilidad}

Lluelles (2010) menciona que la accesibilidad web se refiere a la capacidad de acceso a la información contenida en la red que tiene cualquier persona, independientemente de sus limitaciones físicas, capacidades intelectuales, condiciones ambientales y limitaciones tecnológicas. Las pautas de accesibilidad para contenido web denominadas WCAG 2.0 (Web Content Accessibility Guideline) utilizan cuatro principios: 
- El contenido debe ser perceptible

- La interfaz y sus elementos deben ser operables

- El contenido y los controles deben ser comprensibles

- El contenido debe ser suficientemente robusto como para poder funcionar tanto con la tecnología web actual como con la futura

Griol, Callejas y López (2013) explican los cuatro principios de la accesibilidad de los contenidos audiovisuales:

1. Perceptible: la información y los componentes de la interfaz debe ser presentada a los usuarios en diversas formas

2. Operable: los elementos que conforman la interfaz debe ser sencillos

3. Comprensible: los contenidos y las operaciones de la interfaz debe ser entendible para los usuarios

4. Robusto: El contenido y la interfaz deben ser empleadas por el mayor número de personas posibles

Para cumplir con los criterios relacionados con la accesibilidad del WCAG 2.0, el SUAEB se apoya en el lenguaje HTML5 para ofrecer flexibilidad en los navegadores web y sistemas operativos que emplea el usuario. Además las alternativas textuales junto con el Diseño Gráfico permiten diseñar contenidos audiovisuales perceptible, operable y comprensible. La figura 5 muestra un ejemplo de los contenidos audiovisuales del SUAEB. 
Figura 5. Ejemplo de un contenido audiovisual en el SUAEB.

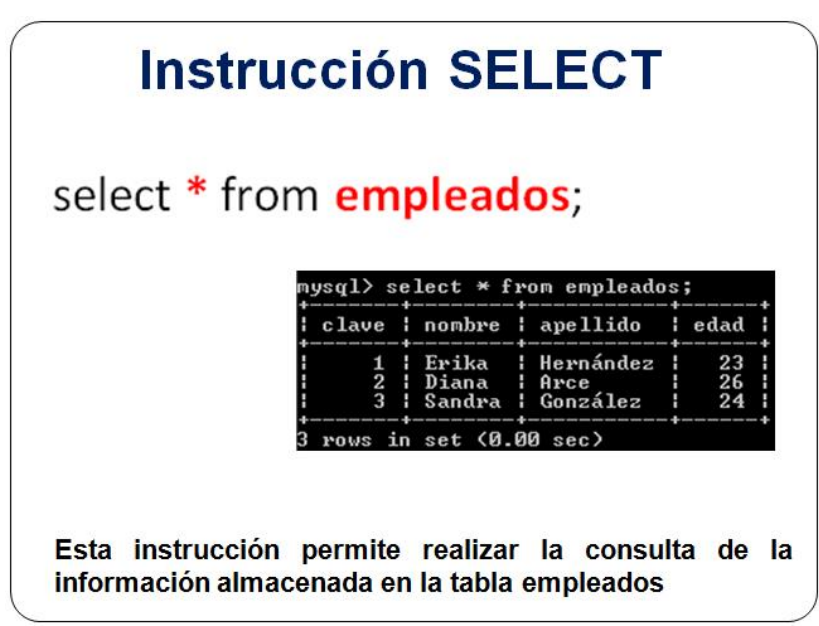

Fuente: Diseño propio, 2015.

En la siguiente sección se explica el enfoque, muestra y las hipótesis utilizadas para esta investigación relacionada con el uso del SUAEB durante el proceso de enseñanzaaprendizaje. 


\section{Resultados}

La figura 6 muestra la arquitectura del SUAEB.

Figura 6. Arquitectura del SUAEB. Diseño propio, 2015.

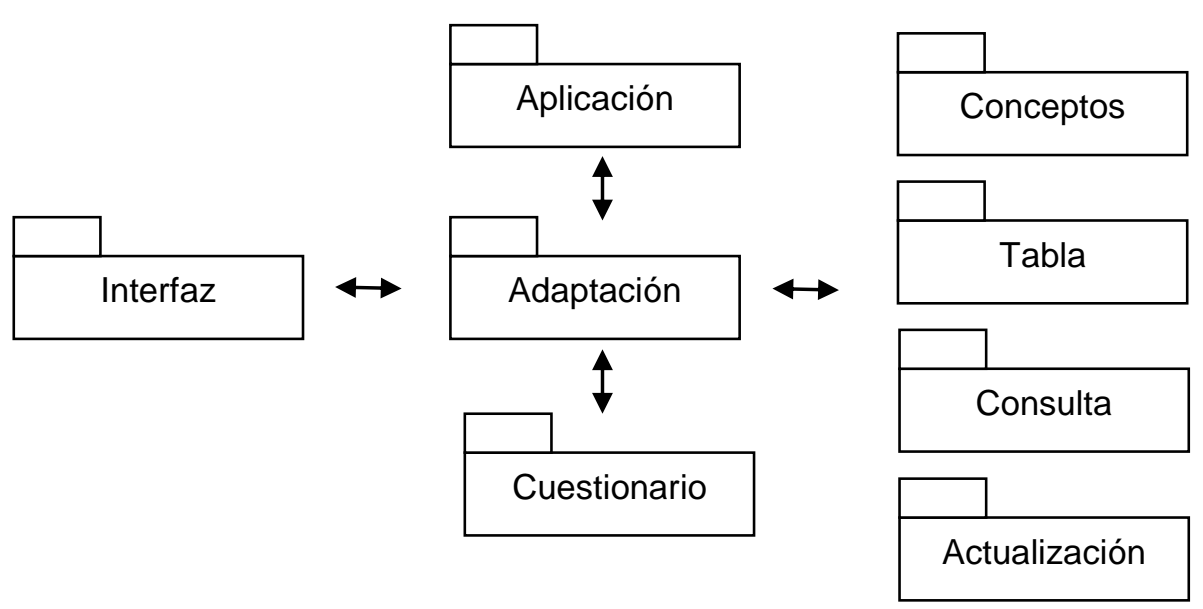

Fuente: Diseño propio, 2015.

A continuación se explican las funciones de los módulos que conforman la arquitectura del SUAEB:

- Módulo de adaptación: Es el encargado de controlar los contenidos audiovisuales sobre las bases de datos por medio de los datos proporcionados por el cuestionario

- Módulo de cuestionario: Consta de 20 preguntas que determinan el conocimiento que necesita el usuario. Las preguntas consideran los temas sobre los conceptos básicos de las bases de datos y las instrucciones de SQL para administrar la información 
- Módulo de conceptos: Contiene la información relacionada con base de datos, los elementos de la tabla, los campos, los registros y la importancia del Lenguaje SQL

- Módulo de tabla : Contiene la información sobre las instrucciones de SQL para administrar la tabla

- Módulo de consulta: Contiene la información sobre las instrucciones de SQL para consultar los datos

- Módulo de actualización: Contiene la información sobre las instrucciones de SQL para actualizar y eliminar los datos

- Módulo de aplicación: Contiene la información sobre un ejercicio que integra las instrucciones de SQL

- Módulo de interfaz : Permite la interacción entre el SUAEB y el estudiante 
La tabla 2 muestra las alternativas para personalizar los contenidos relacionados con el módulo conceptos.

Tabla 2. Contenidos personalizados del módulo conceptos

\begin{tabular}{|c|c|c|c|c|c|}
\hline $\begin{array}{l}\text { Contenidos } \\
\text { Audiovisuales }\end{array}$ & Base de datos & Tabla & Campo & Registro & SQL \\
\hline 1 & & & & & $\mathrm{Si}$ \\
\hline 2 & & & & $\mathrm{Si}$ & \\
\hline 3 & & & & $\mathrm{Si}$ & $\mathrm{Si}$ \\
\hline 4 & & & $\mathrm{Si}$ & & \\
\hline 5 & & & $\mathrm{Si}$ & & $\mathrm{Si}$ \\
\hline 6 & & & $\mathrm{Si}$ & $\mathrm{Si}$ & \\
\hline 7 & & & $\mathrm{Si}$ & $\mathrm{Si}$ & $\mathrm{Si}$ \\
\hline 8 & & $\mathrm{Si}$ & & & \\
\hline 9 & & $\mathrm{Si}$ & & & $\mathrm{Si}$ \\
\hline 10 & & $\mathrm{Si}$ & & Si & \\
\hline 11 & & $\mathrm{Si}$ & & $\mathrm{Si}$ & $\mathrm{Si}$ \\
\hline 12 & & $\mathrm{Si}$ & $\mathrm{Si}$ & & \\
\hline 13 & & $\mathrm{Si}$ & $\mathrm{Si}$ & & $\mathrm{Si}$ \\
\hline 14 & & $\mathrm{Si}$ & $\mathrm{Si}$ & Si & \\
\hline 15 & & $\mathrm{Si}$ & $\mathrm{Si}$ & Si & $\mathrm{Si}$ \\
\hline 16 & $\mathrm{Si}$ & & & & \\
\hline 17 & $\mathrm{Si}$ & & & & $\mathrm{Si}$ \\
\hline 18 & $\mathrm{Si}$ & & & $\mathrm{Si}$ & \\
\hline 19 & $\mathrm{Si}$ & & & Si & $\mathrm{Si}$ \\
\hline 20 & $\mathrm{Si}$ & & $\mathrm{Si}$ & & \\
\hline 21 & $\mathrm{Si}$ & & $\mathrm{Si}$ & & $\mathrm{Si}$ \\
\hline
\end{tabular}


Experiencia de la asignatura de bases de datos con la construcción del sistema web personalizado SUAEB. Caso de estudio Ricardo Adán Salas-Rueda

\begin{tabular}{c|c|c|c|c|c}
\cline { 2 - 5 } 22 & $\mathrm{Si}$ & & $\mathrm{Si}$ & $\mathrm{Si}$ & \\
\hline 23 & $\mathrm{Si}$ & & $\mathrm{Si}$ & $\mathrm{Si}$ & $\mathrm{Si}$ \\
\hline 24 & $\mathrm{Si}$ & $\mathrm{Si}$ & & & \\
\hline 25 & $\mathrm{Si}$ & $\mathrm{Si}$ & & & $\mathrm{Si}$ \\
\hline 26 & $\mathrm{Si}$ & $\mathrm{Si}$ & & $\mathrm{Si}$ & \\
\hline 27 & $\mathrm{Si}$ & $\mathrm{Si}$ & & $\mathrm{Si}$ & $\mathrm{Si}$ \\
\hline 28 & $\mathrm{Si}$ & $\mathrm{Si}$ & $\mathrm{Si}$ & & \\
\hline 29 & $\mathrm{Si}$ & $\mathrm{Si}$ & $\mathrm{Si}$ & & $\mathrm{Si}$ \\
\hline 30 & $\mathrm{Si}$ & $\mathrm{Si}$ & $\mathrm{Si}$ & $\mathrm{Si}$ & \\
\hline 31 & $\mathrm{Si}$ & $\mathrm{Si}$ & $\mathrm{Si}$ & $\mathrm{Si}$ & $\mathrm{Si}$ \\
\hline 32 & & & & & \\
\hline
\end{tabular}

Fuente: Diseño propio, 2015. 
Es importante mencionar que cada módulo que contiene la información sobre las bases de datos se puede personalizar de 32 formas distintas. Por lo tanto, el SUAEB presenta 4 módulos donde se almacena la información dando origen a 128 alternativas de los contenidos audiovisuales.

El método ANOVA con los niveles de significancia correspondientes al 0.01 y 0.05 permiten analizar el comportamiento del estudiante antes y después de usar el SUAEB. En la tabla 3, se observa que el valor estadístico del método ANOVA (30.51) es mayor que el valor de $F(4.11)$ con el nivel de significancia de 0.05 . Por consiguiente, la hipótesis nula $(\mathrm{Ho})$ es rechazada y la hipótesis alternativa $(\mathrm{Ha})$ sobre el SUAEB es aceptada.

Tabla 3. Resultados del método ANOVA $\alpha=0.05$

\begin{tabular}{lccccccc}
\hline $\begin{array}{c}\text { Origen de las } \\
\text { variaciones }\end{array}$ & $\begin{array}{c}\text { Suma de } \\
\text { cuadrados }\end{array}$ & $\begin{array}{c}\text { Grados } \\
\text { libertad }\end{array}$ & $\begin{array}{c}\text { Promedio } \\
\text { de los } \\
\text { cuadrados }\end{array}$ & F & Probabilidad & para F \\
\hline Entre grupos & 55.6842105 & 1 & 55.6842105 & 30.5192308 & $3.0043 \mathrm{E}-06$ & 4.11316522 \\
& & & & & & \\
Dentro de los grupos & 65.6842105 & 36 & 1.8245614 & & & \\
\hline
\end{tabular}

Fuente: Diseño propio, 2015

La tabla 4 muestra que el valor estadístico del método ANOVA (30.51) es mayor que el valor estadístico $F(7.39)$ con el nivel significancia de 0.01 . Por consiguiente, la hipótesis alternativa es aceptada. 
Tabla 4. Resultados del método ANOVA $\alpha=0.01$

\begin{tabular}{|c|c|c|c|c|c|c|}
\hline $\begin{array}{c}\text { Origen de las } \\
\text { variaciones }\end{array}$ & $\begin{array}{l}\text { Suma de } \\
\text { cuadrados }\end{array}$ & $\begin{array}{c}\text { Grados } \\
\text { de } \\
\text { libertad }\end{array}$ & $\begin{array}{l}\text { Promedio } \\
\text { de los } \\
\text { cuadrados }\end{array}$ & $\mathrm{F}$ & Probabilidad & $\begin{array}{c}\text { Valor crítico } \\
\text { para } F\end{array}$ \\
\hline Entre grupos & 55.6842105 & 1 & 55.6842105 & 30.5192308 & $3.0043 \mathrm{E}-06$ & 7.39559664 \\
\hline Dentro de los grupos & 65.6842105 & 36 & 1.8245614 & & & \\
\hline
\end{tabular}

Fuente: Diseño propio, 2015

En resumen, los resultados obtenidos por medio del método ANOVA con los niveles de significancia de 0.05 y 0.01 permiten afirman que el SUAEB facilita la transmisión del conocimiento sobre las bases de datos.

\section{Discusión}

Los docentes deben considerar el uso de la tecnología de vanguardia relacionado con el diseño de sistemas web personalizados durante la planeación y organización de los cursos con la finalidad de facilitar la transmisión del conocimiento. En esta investigación, se diseñó e implementó el SUAEB con el propósito de que los estudiantes reciban los contenidos audiovisuales que necesitan. El módulo de adaptación del SUAEB permite la personalización de la información sobre los conceptos de bases de datos y las instrucciones de SQL.

Para crear el contenido audiovisual, el módulo de adaptación utiliza los datos proporcionados por el cuestionario. Es importante señalar que la información que se personaliza sobre las bases de datos son: Conceptos, Tabla, Consulta y Actualización, 
los cuales pueden ser presentados en 32 formas distintas cada uno, dando origen a 128 contenidos audiovisuales.

Asimismo la usabilidad y la accesibilidad tienen un papel primordial durante la construcción del SUAEB debido a que permiten una navegación fácil, sencilla, intuitiva y rápida en la interfaz web.

Por medio del Método ANOVA con el nivel de significancia de 0.05 y 0.01 , esta investigación acepta la hipótesis alternativa. Por consiguiente, se afirma que la arquitectura del SUAEB mejora el rendimiento académico de los estudiantes. Por esta razón, se sugiere crear sistemas web usables que consideren durante el diseño el nivel del conocimiento que presentan los estudiantes. Se recomienda que en la planeación y organización de los sistemas se consideren los criterios de la usabilidad y los principios de la accesibilidad debido a que estos factores permiten construir una interfaz web útil para los usuarios.

\section{Referencias}

Anderson, D., Sweeney, D. y Williams, T. (2011). Estadística para negocios y economía. Distrito Federal: CENGAGE Learning.

Bernárdez, M. L. (2007). Diseño, producción, implementación de e-learning. Estados Unidos: Global Business Press

Brinck, T., Gergie, D. y Wood, S. D. (2002). Usability for the Web. Estados Unidos: Academic Press

Hepp K., Prats, M. A. y Holgado, J. (2015). Formación de educadores: la tecnología al servicio del desarrollo de un perfil profesional innovador y reflexivo. RUSC. Universities and Knowledge Society Journal, 12(2). 30-43. doi: http://dx.doi.org/10.7238/rusc

González, I. M. y Blanco, L. J. (2012). Sistemas de hipermedia adaptativa en un entorno educativo. Revista COFIN Habana. 2(2), 62-71. (DOI: http://www.cofinhab.uh.cu/index.php/cofin/article/viewFile/64/64) 
Granollers, T., Lorés, J. y Cañas Delgado, J.J. (2005). Diseño de sistemas interactivos centrados en el usuario. España: UOC

Griol, D., Callejas, Z. y López, R. (2013). Technologies for inclusive education: Beyond traditional integration approaches. Estados Unidos: IGI Global

Gross, B. (2011). Evolución y retos de la educación virtual construyendo el e-learning del Siglo XXI. España: UOC

Kazanidis, I. y Satratzemi, M. (2007). Adaptivity in a SCORM Compliant Adaptive Educational Hypermedia System. En L. Howard, L. Frederick, L. Ryson y L. Qing (Eds.), Advances in Web based learning. (pp. 363-374). Alemania: SpringerVerlag.

Kendall, K. y Kendall, J. (2011). Análisis y diseño de sistemas. México: Pearson.

Lawrence, D. y Tavakol, S. (2007). Balanced website design. Optimising aesthetics, usability and purpose. Reino Unido: Springer

León, A. (2008). Enterprise Resource Planning. Nueva Delhi: McGraw-Hill

Llanos, D. R. (2007). Fundamentos de informática. España: Paraninfo

Lluelles, M. (2010). Accesibilidad web, una realidad en el aula. En C. Barba y S. Capella (Eds), Ordenadores en el aula. (pp. 255-265). España: Graó

Ramos , A. y Ramos, M. J. (2007). Operaciones con bases de datos ofimáticas y corporativas.España: Thomson

Rubin, J. y Chisnell, D. (2008). Handbook of Usability testing. How to plan, design and conduct effective tests. Estados Unidos: Wiley

Ruiz , E. (2007). Innovación en el aprendizaje de las ciencias y la tecnología. España: Díaz de Santos

Salaverría, R. (2005). Cibermedios: el impacto de Internet en los medios de comunicación en España. Madrid: Comunicación Social.

Suárez, M. C. (2011). Sirus: Sistemas de evaluación de la usabilidad web orientado al usuario y basado en la determinación de tareas críticas. Tesis de Doctorado, Universidad de Oviedo.

Vázquez, J. (2015). Nuevos escenarios y tendencias universitarias. Revista de Investigación Educativa, 33(1), 13-26. Recuperado en: http://revistas.um.es/rie/article/view/211501/172421 